\title{
Research on Factors and Strategies of Knowledge Transfer within Scientific Research and Innovation Teams in Universities
}

\author{
Guizhu Xie ${ }^{1}$, Jianchao Yang $^{2}$, Ying Tao ${ }^{3}$ \\ ${ }^{1)}$ School of Economics and Management, Jiangsu University of Science and Technology, Zhenjiang, Jiangsu, China \\ ${ }^{2)}$ School of National Defense, Jiangsu University of Science and Technology, Zhenjiang, Jiangsu, China \\ ${ }^{3)}$ China Leihuaelectronic Technology Researchinstitute, Wuxi, Jiangsu, China
}

\begin{abstract}
Combining the constructs of organization with knowledge transfer process, we analyze the influential factors of knowledge transfer within scientific research and innovation teams in universities from five aspects: the knowledge source and the knowledge recipients, the knowledge transferred, the context of transfer and the medium of transfer. Based on this study, the paper provides an influential factor model and management strategies for the internal knowledge transfer of the scientific research and innovation team in order to give some guidelines and conferences for the construction of scientific research and innovation teams in universities.
\end{abstract}

Key words - scientific research and innovation teams in universities, knowledge transfer, influential factors, strategies

\section{高校科研团队内部知识转移影响因素及其对策研究}

\author{
谢贵柱 $^{1}$ 杨建超 $^{2}$ 陶莹 $^{3}$ \\ 1) 江苏科技大学经济管理学院, 镇江, 江苏, 中国 \\ 2) 江苏科技大学国防学院，镇江，江苏，中国 \\ 3) 中国雷华电子技术研究所, 无锡, 江苏, 中国
}

摘 要 结合高校科研团队组织结构和知识转移过程, 从知识源特性、知识接收者特性、知识特性、转移环境和转移媒介五个主 要角度探讨了高校科研团队内部知识转移的影响因素, 在此基础上构建了影响因素理论模型, 提出了高校科研团队内部知识转移的管 理对策, 以期能为高校科研团队的建设提供指导与参考。

关键词 高校科研团队, 知识转移, 影响因素, 对策

\section{1. 引言}

十八大报告指出: “完善知识创新体系, 强化基础研究、 前沿技术研究、社会公益技术研究, 提高科学研究水平和 成果转化能力, 抢占科技发展战略制高点。”在当今知识经 济社会里, 组织的存在与发展越来越需要知识的支撑。高

教育部人文社会科学研究规划基金项目“高校创新性科研团队知 识转移影响因素实证研究”(编号:12YJA630169);

江苏省高校哲学社会科学研究项目 “大学生科研创新团队内部知 识转移影响因素实究”(编号:2011SJD880114);

江苏省“十二五”高等教育科学研究规划课题“高校科研创新团队 内部知识转移影响因素实证研究”(编号: KT2011044)
校作为知识型的社会组织, 其在社会发展、国家创新的过 程中发挥着弥足轻重的作用。科研项目所涉知识繁杂程度 的增加, 规模的扩大以及多学科之间的相互交叉融合, 成 为知识创新愈发依赖团队化科研作战的主要原因, 科研团 队已成为高校科学研究的基本组织形式。团队内部的知识 转移是高校科研团队的主要特征之一, 是团队知识创新的 源泉, 团队内部知识转移的绩效直接影响着团队知识创新 的能力 [1]。高校科研团队知识转移是当前国内外学术界关 注的热点问题, 然而由于受到许多内外因素的影响, 知识 转移绩效往往较低。本研究将在识别高校科研团队内部知 识转移关键影响因素的基础上, 为高校科研团队的知识管 理提出有效的管理对策。 


\section{2. 高校科研团队与知识转移}

知识型团队 (Knowledge-Based Workteam) 是指以具有 较高知识背景和创新能力的知识型员工为主体构成、主要 以任务为导向的团队 [2]。高校科研团队是知识型团队中的 一种, 即由科研目标相同，技能互补，具有良好创新意识 及团队合作精神的科研人员所组成的从事相关研究的团体 组织。它主要依托攻关课题、专门研究中心、省级以上重 点实验室、相关科技创新平台来开展研究工作。一般由团 队负责人、科研骨干、一般科研（辅助）成员组织。

1977 年, 美国技术创新管理学家 TEECE 首次提出知 识转移的思想, 他主张技术进行国际转移能够帮助企业积 累有价值的知识，促进技术扩散与应用，缩小不同地区间 的技术差距 [3]。之后, 知识转移的研究受到越来越多国内 外学者的关注。Blood good \& Salisbury[4]认为知识转移是 指知识通过不同的形式, 在不同的个体间、组织间、个体组织间实现传递、转移的过程。卫洁等[5]认为知识转移是 一个开放的系统, 它与外部环境存在着信息流和能量流的 交换, 知识转移系统中各主体间的相互作用和联系促使系 统产生单个主体所不具备的功能特性。

高校科研团队内部知识转移可以实现不同学科知识的 传播、交流与吸收, 增加知识个体存量, 最大限度的汇集 知识源; 在拓展团队整体知识面的同时可以促进知识创新, 完成团队科研目标。

\section{3. 高校科研团队内部知识转移影响因素}

本文在对现有研究成果和专家访谈结果详细分析的基 础上, 结合高校科研团队自身的特点, 对其内部影响因素 进行了详细的梳理和深入的思考。最终得出: 其主要受到 知识源特性、知识接收者特性、知识特性、转移环境和转 移媒介五个主要方面因素的影响。

\section{1 知识源特性}

(1) 转移意愿

转移意愿体现知识所有者对自身知识转移的意愿程 度, 其强弱程度会直接影响到知识转移的数量与质量, 如 果缺乏知识转移意愿, 知识转移就会很难成功。Anna Wiewiora[6]认为转移意愿越强, 越有利于知识的分享和转 移。知识作为一种重要的财富资源, 在高校科研团队内部, 部分成员可能出于对信任机制的担心、知识优势的保护及 利益诱因的不足, 会降低其转移的意愿, 从而导致知识转 移绩效的下降。

(2) 传递能力

传递能力是指知识转移者知道如何运用自身知识, 并
把知识传递给另一方的能力。知识传出者作为知识源的发 送方, 其具备的技能、经验与沟通编码能力, 将会影响到 接受者对其理解、吸收的程度。知识源的传递能力越强, 知识转移的效果越好。由于高校科研团队内部成员所具备 的知识不尽相同, 需要采取不同的转移方式。因此, 知识 源的转移能力势必会影响到其转移效果。

\section{2 知识接收者特性}

(1) 接收意愿

接收意愿是指知识接收方接收知识和主动参与知识转 移过程的意愿程度, 对知识转移是否顺利进行起到直接的 影响。企业员工的学习热情越高, 越能提高知识转移的效 率 [8]。知识的接受与学习, 是一个需要耗费大量精力与时 间的过程; 出于对接受新知识的心理负担、知识转移预期 不高、“面子”问题及缺乏内外激励刺激等原因, 知识接受 者会降低自己接受知识的意愿, 采取消极的态度, 不自觉 的设置屏障, 从而阻碍知识的吸收与保持。

(2) 吸收能力

吸收能力是指知识接受者接受、吸收、消化新知识, 通过应用而转化为自身知识的能力。知识转移的效果不仅 是由知识转移能力决定的, 而且受知识接受者的吸收能力 影响。吴传荣等[7]指出企业的知识吸收能力越高, 利用网 络中的公共知识或企业原有知识的能力就越强, 创新能力 提高越快, 创造的利润越高。知识接收方具有较强的吸收 能力, 能够快速明确所需要的知识并锁定知识源, 从而提 高获取知识的速度与效率，加快新知识的消化及应用。

\section{3 知识特性}

(1) 隐含性

Polanyi 最早提出知识的隐性(tacitness)特质, 并根据知 识的可表达程度将知识分为显性知识与隐性知识 [8]。显性 知识是指人们能够通过文字、图像、科学公式和系统语言 加以表达且容易储存, 受时间、空间影响小的知识。隐性 知识则是难以通过具体的符号进行描述和表达的知识, 它 往往来源于人们的经验与技能, 需要通过感觉和接触才能 获知, 具有不易编码、难以传授等特征。显性知识能够通 过具体的信号或符号表达出来, 通俗易懂, 利于知识的传 递; 而隐性知识带有较强的模糊性, 不易编码与理解, 传 递难度大。知识的隐含性越高, 需要付出的努力就越多, 就越不利于知识的转移。

(2) 复杂性 知识复杂性指特定知识所涉及到的相互依赖的技术、 
路线、人员、知识源的个数较多, 根据复杂性高低, 知识 可以划分为简单知识与复杂知识。简单知识容易理解和表 达, 转移难度低, 而复杂知识不易理解和传输, 转移难度 大。高校科研团队内部成员由于自己的专业、知识结构和 学科背景不尽相同, 在一定程度上增加了团队整体知识的 复杂度, 影响知识转移的效果。当知识复杂性高时, 知识 距离加大转移难度的效果增强; 当知识复杂性低时, 知识 距离加大转移难度的效果减弱 [9]。

\section{4 转移环境}

(1) 团队文化

团队文化是指团队成员在相互合作的过程中, 为实现 自我价值而普遍遵守和奉行的共同价值观念, 及在此过程 中所营造出的一种团队氛围与团队精神的潜意识文化。团 队文化反映了团队成员对待问题的态度与习惯, 影响成员 学习、工作的氛围与热情。营造良好的团队文化, 增强团 队的凝聚力、向心力, 有利于激发成员的工作积极性, 形 成统一的思想与行动, 促使团队成员为共同的目标而奋斗。 因此, 团队文化对知识转移起到正相关的作用。

\section{(2) 信任关系}

信任关系反映了团队成员之间的亲密程度, 是良好人 际关系的基础。交流的基础是信任, 信任在隐性知识与复 杂知识的转移中发挥着重要的作用, 只有相互信任, 交流 双方才能打开心扉, 实现知识的交流。团队内成员间关系 的紧密程度和知识转移的环境, 是影响知识转移效果的重 要因素。社会交往联系既能促进信任, 提高感知的可信度, 信任和可信度又会促进更多的资源交换 [10]。因此, 团队成 员间的信任有利于知识交流, 提高知识转移绩效。

(3) 激励机制

激励机制是组织运用特定的方法与制度, 去调动员工 的积极性和创造性, 实现组织预期任务与目标的过程。有 效的激励可以满足个体需求, 激发员工积极性, 并有效的 实现知识共享, 同样也成为高校创新性科研团队吸引、留 住高层次人才并实现人才高效利用的关键所在。良好的激 励机制有利于高校科研团队实现知识创新, 而激励措施的 缺少, 会使知识发送方因担心知识共享后得不到相应的回 报, 而对自身知识予以保护, 从而降低知识转移的积极性, 最终导致绩效的下降。

\section{5 转移媒介}

转移媒介是知识源与知识接受者之间传递知识的桥 梁, 从结构角度看, 转移媒介由知识编码和转移通道构成
[11]。知识编码可以表明知识的表述方式, 而知识隐含性、 复杂性的特点决定了知识需要不同的传递工具和手段, 配 适相应的转移通道。转移渠道往往还会受到知识转移意愿、 能力、环境等因素的影响。合适的转移渠道或媒介, 有利 于提高知识转移的效果。高校创新性科研团队在进行内部 知识转移时, 要充分考虑到知识转移过程的方方面面, 选 择正确的知识转移媒介, 从而实现知识的内化和外化。

\section{4. 促进高校科研团队内部知识转移的应对策略}

通过对上述相关因素的分析, 构建了高校科研团队内 部知识转移影响因素模型(如图 1 所示), 并提出了高校科研 团队内部在知识转移中应采取的应对策略。



图 1 高校科研团队内部知识转移影响因素模型

\section{1 构建合理的团队组织结构}

通过上述相关因素的分析及高校科研团队内部知识转 移影响因素模型可见, 知识隐含性、复杂性与知识转移绩 效成负相关, 传递能力、吸收能力与知识转移绩效成正相 关。因此, 如何降低知识的隐含性和复杂性, 提升传递能 力和吸收能力, 成为提高高校科研团队知识转移绩效的关 键之处。

高校科研团队往往由不同学科、不同知识领域的专家 与学者组成, 团队领导作为团队的学术带头人, 在团队中 发挥着至关重要的作用。高校科研团队在吸纳不同知识背 景的专家与学者时, 要充分考虑团队学历结构和知识结构 的科学性、合理性, 创造隐性知识的共享环境, 实现隐性 知识到显性知识的转化, 从而提高知识转移的绩效。同时, 通过加强培训, 引入有效的学习方法, 加强知识双方沟通 能力和表达能力的训练, 鼓励成员进行交流、深度对话, 增加成员的知识存量, 提高团队内部知识的吸收能力。 


\section{2 创建坦诚互信的团队文化}

通过上述相关因素的分析及高校科研团队内部知识转 移影响因素模型可见, 团队文化、信任关系与知识转移绩 效成正相关, 即团队文化的氛围越好、信任关系越强, 知 识转移的效果越好。

团队文化的营造是一个漫长的过程, 信任是知识转移 的基础, 是营造良好团队文化的关键。这需要团队创造宽 松的交流环境, 构建知识共享机制, 营造争鸣式学习氛围, 创建适合团队交流的知识转移通道。通过不断发挥团队领 导者的模范作用, 利用俱乐部、周末沙龙类似的活动加强 成员沟通与交流, 增进团队成员的信任、理解与认同, 从 而增进感情, 消除和化解各种影响知识传递的文化和沟通 障碍, 提高团队的凝聚力, 营造良好的团队文化, 实现知 识的有效转移。

\section{3 完善激励考核机制, 培养成员的创新意识}

通过上述相关因素的分析及高校科研团队内部知识转 移影响因素模型可见, 转移意愿、接受意愿、激励机制与 知识转移绩效成正相关, 即转移意愿、接受意愿越强, 激 励的方式与措施越科学, 知识转移的效果越好。

高校科研团队在进行科研工作, 完成一项科研项目时, 不仅需要物质的投入, 还需要科研人员在时间、精力的大 量投入和充分的学术交流。团队成员的工作态度、积极性 与创新意识在这一过程中显得就尤为重要, 这需要团队做 好相应的激励措施, 提高双方的转移意愿和接受意愿。高 校科研团队内部应建立科学、合理的激励考核机制：一方 面坚持物质奖励、精神奖励相结合，使其真正体现出科研 工作的价值与特点, 不要盲目的追究成果, 更应该注重科 研的质量和科研工作者的贡献，毕竟科研工作是一个探索 的过程, 成果产出所需的时间存在不确定性。另一方面要 实行团队考核成绩与个人考核成绩相结合的策略, 预防和 杜绝“搭便车”的现象，提高团队集体荣誉感和合作精神， 提升团队的整体绩效。

\section{4 选择恰当的转移媒介}

通过上述相关因素的分析及高校科研团队内部知识转 移影响因素模型可见, 知识转移媒介对知识转移绩效有重 要影响, 即知识转移媒介越恰当, 知识转移绩效越好。

书面交流方式是指知识传输者通过文字、图像、专业 符号、数学公式及程序代码等书面表达的形式将知识传递 给接收方。显性知识易于表达且容易编码, 适合采用书面 式的传递方式。面对面的交流方式是指人们在面对面的基
础上, 通过错综复杂的声调、肢体语言, 以增加彼此亲密的 接触、交流的信服力, 从而实现交流的目的。隐性知识由 于不易编码、模糊程度高, 面对面的交流是其最优转移媒 介。同时，随着信息技术的发展，其在提高工作任务和流 程的结构化、规范化和自动化程度, 节约时间, 提高效率 方面上发挥着重要的作用。在以后的知识转移过程中，应 充分发挥信息技术在知识的获取、储存、转移和创造过程 中的作用, 更要利用其在显性知识转化、数据挖掘、信息 检索、决策支持等方面的优势, 提高高校科研团队知识转 移绩效。

\section{参考文献(References)}

[1] Haitao Zhang, Yanling Wu. Research on Internal Knowledge Transfer Mechanism of College Science Research Teams Based on Knowledge Energy. Library and Information Service, 2010, 54(20): 110-114.

[2] Yahong Li, Jian Li.The Performance Incentive Mechanism Research and Case Analysis of Knowledge-based Workteam. Technoeconomics \& Management Research, 2011, (10):50-53.

[3] Teece D J, Pisano G, Shuen A. Dynamic capabilities and strategic management. Strategic management journal, 1997, 18(7): 509-533.

[4] Blood good JM, Salisbury WD. Understanding the influence of organizational change strategies on information technology and knowledge management strategic. Decision Support Systems, 2001, (31):55-69.

[5] Jie Wei, Chonghuai Niu. The Modeling and Simulation of Knowledge Transfer System under the Technology Talents Aggregation. Science \& Technology Progress and Policy, 2013, 30(5):116-121.

[6] Lei Nie. Reflections upon Knowledge Transfer in Cross-Border Mergers and Acquisitions of Chinese Enterprises. Science \& Technology Progress and Policy, 2012, 29(12):151-155.

[7] Chuanron $\mathrm{Wu}$, Yingwu Chen. Time optimization of knowledge transfer in high - tech enterprises innovation networks. Systems Engineering - Theory \& Practice, 2013, 33(4): 955-962.

[8] POLANYI M. Personal knowledge: toward a post critical philosophy. Chicago; University of Chicago Press, 1992.

[9] Yongxing Wang, Xiping Zhao, Mi Zhou, Yuanmei Qu. The Moderating Effect of Knowledge Characteristics and Interpersonal Trust in the Knowledge Transfer Process. Soft Science, 2012, 26(9):24-29.

[10] Kunpeng Yu, Dongqiang Guo. Research on the Knowledge Transfer of Transformation Business Based on System Dynamics. Soft Science, 2013, 27(3):111-115,139.

[11] Yanling Zhang. Knowledge Transfer Path Analysis of Agricultural Brainpower Based on KTA Framework. Journal of Anhui Agricultural Sciences, 2013, (2):882-883,892. 